\title{
Synthesis and Characterization of Nanocrystalline Zinc Oxide Thin Films for Ethanol Vapor Sensor
}

\author{
Bhasha $\mathbf{S}^{1 *}$, Malik $\mathbf{P}^{1}$, Santosh $\mathbf{S}^{2}$ and Purnima $\mathbf{J}^{1}$ \\ ${ }^{1}$ Department of Chemistry, Netaji Subhas Institue of Technology, Dwarka, New Delhi, India \\ ${ }^{2}$ CSIR-National Physical Laboratories, New Delhi, India
}

\begin{abstract}
$\mathrm{ZnO}$ nanoparticles as gas sensors have a property of changing the electrical conductivity of the sensing element on exposure to gas atmospheres. The number, density and size distribution of particles plays a key role in gas sensing applications. The gas sensing mechanism mainly depends upon the morphology, ion occupancy and the operating temperature, which helps in adsorbing the $\mathrm{OH}$ - groups on the surface of oxide materials. The ethanol vapor sensing measurements have been carried out on the $\mathrm{ZnO}$ pellet with silver pasted electrodes on both the ends. The change in electrical resistance was used as the measure of ethanol vapor response at different temperatures in the range of $25^{\circ} \mathrm{C}$ to $45^{\circ} \mathrm{C}$ at an interval of $5^{\circ} \mathrm{C}$ under an ethanol concentration of $25.02,50.05,75.08,100.11$ and $150.17 \mathrm{ppm}$. These results showed that the indium zinc oxide film based ethanol sensor has high response quick recovery time and selectivity to the ethanol vapor.
\end{abstract}

Keywords: ZnO; Nano particles; Ethanol gas sensor; X-RD; TEM

\section{Introduction}

Zinc oxide $(\mathrm{ZnO})$ nanoparticles have importance due to their vast area of applications, e.g., gas, chemical and bio-sensors, cosmetics, storage, optical and electrical devices, window materials for displays, solar cells, and drug-delivery [1-5]. $\mathrm{ZnO}$ is an attractive material for short-wavelength opto-electronic applications owing to its wide band gap $3.37 \mathrm{eV}$, large bond strength, and large exciton binding energy (60 $\mathrm{MeV}$ ) at room temperature [6]. As a wide band gap material, In addition, due to its non-centrosymmetric crystallographic phase, $\mathrm{ZnO}$ shows the piezoelectric property $[7,8]$, which is highly useful for the fabrication of devices, such as electromagnetic coupled sensors and actuators. $\mathrm{ZnO}$ have extensive applications in water purification [9]. $\mathrm{ZnO}$ is inexpensive n-type semiconductor [10]. Nanostructures made of $\mathrm{ZnO}$ have attracted significant attention due to their proposed applications in the low voltage and short-wavelength $368 \mathrm{~nm}$ electro-optical devices transparent ultraviolet UV protection films and gas sensors. Therefore, several new routes have been developed to synthesize $\mathrm{ZnO}-\mathrm{NPs}$, such as, sol-gel, sol-gel combustion, chemical precipitation [11,12], hydrothermal, solvothermal, chemical vapor deposition (CVD), a sonochemical method, and thermal oxidation [13-15]. Sol-gel method is widely adopted for the fabrication of transparent and conducting oxide due to its simplicity, safety, no need of costly vacuum system and hence cheap method for large area coating. Next, ethanol sensing property of $\mathrm{ZnO}$ sample (i.e. $\mathrm{ZnO}$ quantum dots embedded in PVP) has been examined by exploring the variation of sample resistance with time in presence of ethanol vapour [7]. Vapour sensing property of semiconductor is purely a surface phenomenon.

The main advantages of chemical sensors are their low price, small size, high sensitivity, and low power consumption. The use of nanostructured materials for the sensing device is envisaged to further improve the sensitivity of these devices. $\mathrm{ZnO}$ morphology could generate a large number of active centers that enhanced the ethanol gas response. Ethanol is one of the most commonly used and widespread alcohols, and thus there is a need to develop sensors for its detection. The most common application of ethanol sensors is as a breath analyzer, since the ethanol vapor inhuman breath is said to be correlated with the concentration in the blood. A semiconductor gas sensor possesses a property of changing the electrical conductivity of the sensing element on exposure to different gas atmospheres. The gas sensing mechanism mainly depends upon the morphology, ion occupancy, the operating temperature, which helps in holding the $\mathrm{OH}$ groups on the surface of oxide materials. One of the requirements of the gas sensors is low power consumption, because the sensors need to work reliably and continuously. A low resistance material has lower driving power when it is used as a sensor. Appropriate donor doping can produce the electronic defects that increase the influence of oxygen partial pressure on the conductivity. The higher operating temperature (approximately $400^{\circ} \mathrm{C}$ ) and poor sensitivity are some of the major concerns in using $\mathrm{ZnO}$ as sensing layer. The $\mathrm{ZnO}$ compound has been considered a potential gas detection device because it exhibits a good chemical sensitivity to different analyte gases, fast response time, and a shorter recovery time.

The optimum working temperature of semiconductor gas sensor depends upon the gas atmosphere and on the properties of the sensing material. Ethanol is one of the most commonly used and widespread alcohols, and thus there is a need to develop new generation sensors for its detection. The most common application of ethanol sensors is as a breath analyzer, since the ethanol vapors in human breath is said to be correlated with the concentration in the blood.

\section{Experimental measurements}

Nanocrystalline (NC) ZnO thin films was grown by sol-gel process [16,17]. Zinc acetate was dissolved in $100 \mathrm{ml}$ zinc acetate as precursor $\left[\mathrm{Zn}\left(\mathrm{CH}_{3} \mathrm{COO}\right)_{2} .2 \mathrm{H}_{2} \mathrm{O}\right]$. The $\mathrm{pH}$ value of the solution was between 3.7 and 4.0. The mixture was heated at $80^{\circ} \mathrm{C}$ under continuous stirring for $3 \mathrm{~h}$ after which precipitate occurred. Then the solution is left for 30 minutes which results in the formation of white bulky solution. The

*Corresponding authors: Bhasha S, Department of Chemistry, Netaji Subhas Institue of Technology, Dwarka, Sec-3, New Delhi, India, Tel: 0112509 9050; E-mail: sharmabhasha@gmail.com

Received June 01, 2015; Accepted June 12, 2015; Published June 22, 2015

Citation: Bhasha S, Malik P, Santosh S, Purnima J (2015) Synthesis and Characterization of Nanocrystalline Zinc Oxide Thin Films for Ethanol Vapor Sensor. J Nanomed Nanotechnol 6: 306. doi:10.4172/2157-7439.1000306

Copyright: @ 2015 Bhasha S, et al. This is an open-access article distributed under the terms of the Creative Commons Attribution License, which permits unrestricted use, distribution, and reproduction in any medium, provided the original author and source are credited. 
solution is then washed 8-10 times with distilled water and filtered in a filter paper. The residue obtained is put for drying in oven at a temperature of about $95^{\circ} \mathrm{C}$ for $8 \mathrm{hr}$. The precipitates were recovered, thoroughly rinsed in distilled water followed by drying wafer at $100^{\circ} \mathrm{C}$ and subsequent annealing at $100^{\circ} \mathrm{C}, 200^{\circ} \mathrm{C}, 300^{\circ} \mathrm{C}$ and $400^{\circ} \mathrm{C}$ for one hour to optimize temperature suitable for good quality $\mathrm{ZnO}$ films. Finally the sensing response of nanorods with nanoparticles of zinc oxide to ethanol at different temperatures has also been compared.

Ten coatings were done to obtain the optimum thickness of the film needed for characterization. The spherical zinc oxide nanoparticles were produced using different concentration of zinc acetate which was used as the zinc source by simple sol gel method which is short time process and cost effective [18]. The film thickness was $250 \mathrm{~nm}, 256 \mathrm{~nm}$ and 334 $\mathrm{nm}$ by elipsometric investigations. The structural, morphological and optical properties of these particles were characterized by XRD, TEM and UV-VIS techniques (Figure 1).

Zinc oxide crystallizes in a wurtzite structure with alternating planes of tetrahedral coordinated $\mathrm{Zn}^{2+}$ and $\mathrm{O}^{2-}$ bonded alternately along c-axis of hexagonal unit cell with $a 0=0.3250 \mathrm{~nm}$ and $c 0=0.5207 \mathrm{~nm}$. The cause for the natural $\mathrm{N}$-type nature of $\mathrm{ZnO}$ is due to the sensitiveness of $\mathrm{ZnO}$ lattice constants to the presence of structural point defects (vacancies and interstitials) and extended defects (threading/planar dislocations) that are commonly found in $\mathrm{ZnO}$ resulting in a non-stoichiometric compound $\mathrm{Zn} 1+\mathrm{d} \mathrm{O}$ with an excess zinc.

The end product was pale white precipitate. The calcinated powders are studied using different characterization techniques. Confirmation of pure $\mathrm{ZnO}$ phase is verified by XRD analysis.

\section{Results and Discussion}

\section{XRD pattern}

Synthesized NC-ZnO particles are analyzed on the basis of their crystallinty, crystallite size, band gap and structural properties. X-Ray Diffraction pattern (Figure 2) is used to calculate crystallite size and variation in band gap. Unique characteristic X-ray diffraction pattern of each crystalline solid gives the designation of "fingerprint technique" to XRD for its identification. The XRD of the annealed film shows the peaks corresponding to $\mathrm{ZnO}$, confirming that the $\mathrm{Zn}$ film has been completely oxidized to form $\mathrm{ZnO}$.

The XRD pattern consists of a single (002) peak which occurred due to $\mathrm{ZnO}$ crystals and grows along the c-axis. High purity and crystallinity of the prepared $\mathrm{ZnO}$ NPs confirms the sturdy and clear peak. For other

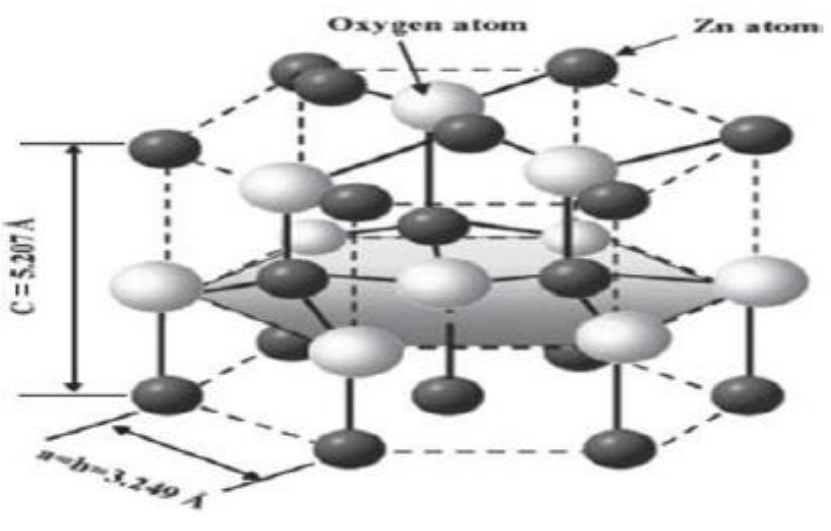

Figure 1: Structure of $\mathrm{ZnO}$. impurities no characteristic peak was accessible [19]. The deviation of the lattice parameters is caused may be due to presence of various point defects such as zinc antisites, oxygen vacancies, and extended defects, such as threading dislocation. With increasing calcinations temperature peak height increases and FWHM decreases as result diffraction peaks become stronger and sharper, thereby indicating that the crystal quality has been improved and the size of particles become bigger [20-24]. Moreover, all diffraction peaks of the product show stronger peak intensities, indicating that the obtained $\mathrm{ZnO}$ nanoparticles have high crystallinity.

\section{EDX and UV-VIS spectra}

The EDX of the $\mathrm{ZnO}$ sample was done by the SEM machine. The EDX reveals that the required phase has present. Both Zinc (Zn) and Oxygen $(\mathrm{O})$ is present in the sample. Again the graph shows the presence of $\mathrm{ZnO}$ nanoparticles. This is due to the substrate over which it was held to do the SEM characterization. Energy dispersive X-ray spectroscopy (EDS, EDX or EDXRF) is an analytical technique used for the elemental analysis or chemical characterization of a sample.

The size of the $\mathrm{ZnO}$ nanoparticles plays an important role in changing the entire properties. Thus, size evolution of semiconducting nanoparticles becomes very essential to explore the properties of the materials. UV-visible absorption spectroscopy is widely being used technique to examine the optical properties of nanosized particles. The absorption spectrum of $\mathrm{ZnO}$ nanopowder is shown in Figure 3. It exhibits a strong absorption band at about $355 \mathrm{~nm}$. An excitonic absorption peak is found at about $258 \mathrm{~nm}$ due to the $\mathrm{ZnO}$ nanoparticles which lie much below the band gap wavelength of $358 \mathrm{~nm}$.

\section{Resistance vs. time of EtOH sensor}

The gas sensing mechanisms normally accepted for semiconductor sensors assume that the oxygen adsorbed on the surface of the oxide removes some of the electronic density and thus decreases the material's conductivity. When reduction gas molecules come into contact with this surface, they may interact with this oxygen, leading to an

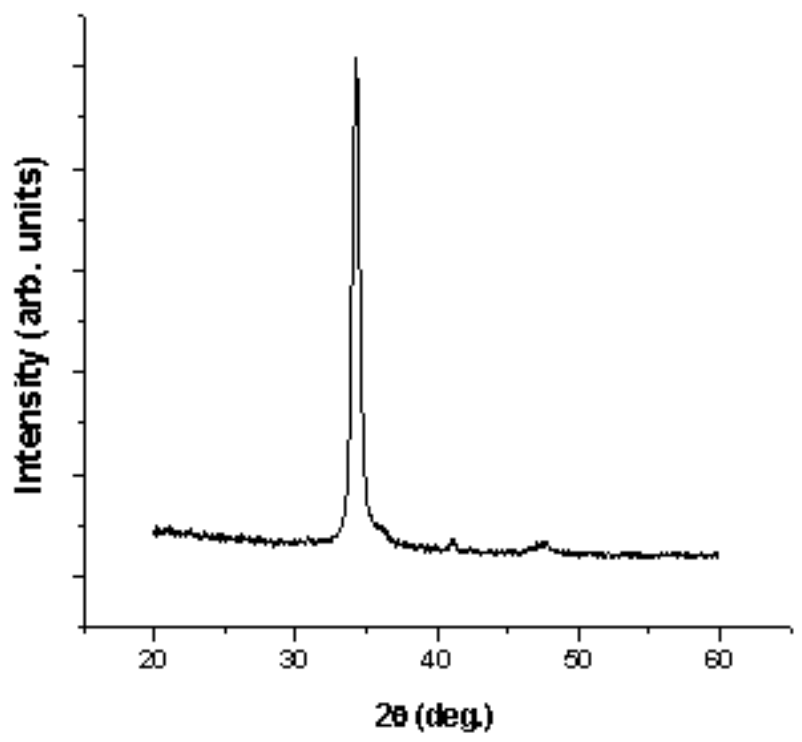

Figure 2: ZnO thin film by XRD pattern. 
Citation: Bhasha S, Malik P, Santosh S, Purnima J (2015) Synthesis and Characterization of Nanocrystalline Zinc Oxide Thin Films for Ethanol Vapor Sensor. J Nanomed Nanotechnol 6: 306. doi:10.4172/2157-7439.1000306

Page 3 of 4

inverse charge transference [20]. Upon the return of the electrons to the conduction band, conductivity increases. This utilizes the gas-induced resistance variations in potential barrier height at grain boundaries (i.e. changes in thickness of the space charge layer) to detect ethanol vapors in air (Figure 4).

The change in electrical resistance was used as the measure of ethanol vapor response at different temperatures in the range of $25^{\circ} \mathrm{C}$ to $45^{\circ} \mathrm{C}$ at an interval of $5^{\circ} \mathrm{C}$ under an ethanol concentration of $25,50,75,100$ and $150 \mu \mathrm{L}$. The possibility of a reaction of ethanol with the $\mathrm{ZnO}$ nanoparticles sensing layers can be explained as two oxidation states [21-22]:

$\mathrm{C}_{2} \mathrm{H}_{5} \mathrm{OH}(\mathrm{g})+[\mathrm{O}] \rightarrow \mathrm{CH}_{3} \mathrm{CHO}+\mathrm{H}_{2} \mathrm{O}$ (the dehydrogenation to acetaldehyde)

$$
\mathrm{C}_{2} \mathrm{H}_{5} \mathrm{OH}(\mathrm{g})+[\mathrm{O}] \rightarrow \mathrm{C}_{2} \mathrm{H}_{4}+\mathrm{H}_{2} \mathrm{O} \text { (the dehydration to ethylene) }
$$

in which $[\mathrm{O}]$ represents the surface oxygen ions.

The first reaction is a process initiating the oxidation by the dehydrogenation to $\mathrm{CH}_{3} \mathrm{CHO}$ intermediate, and the second reaction

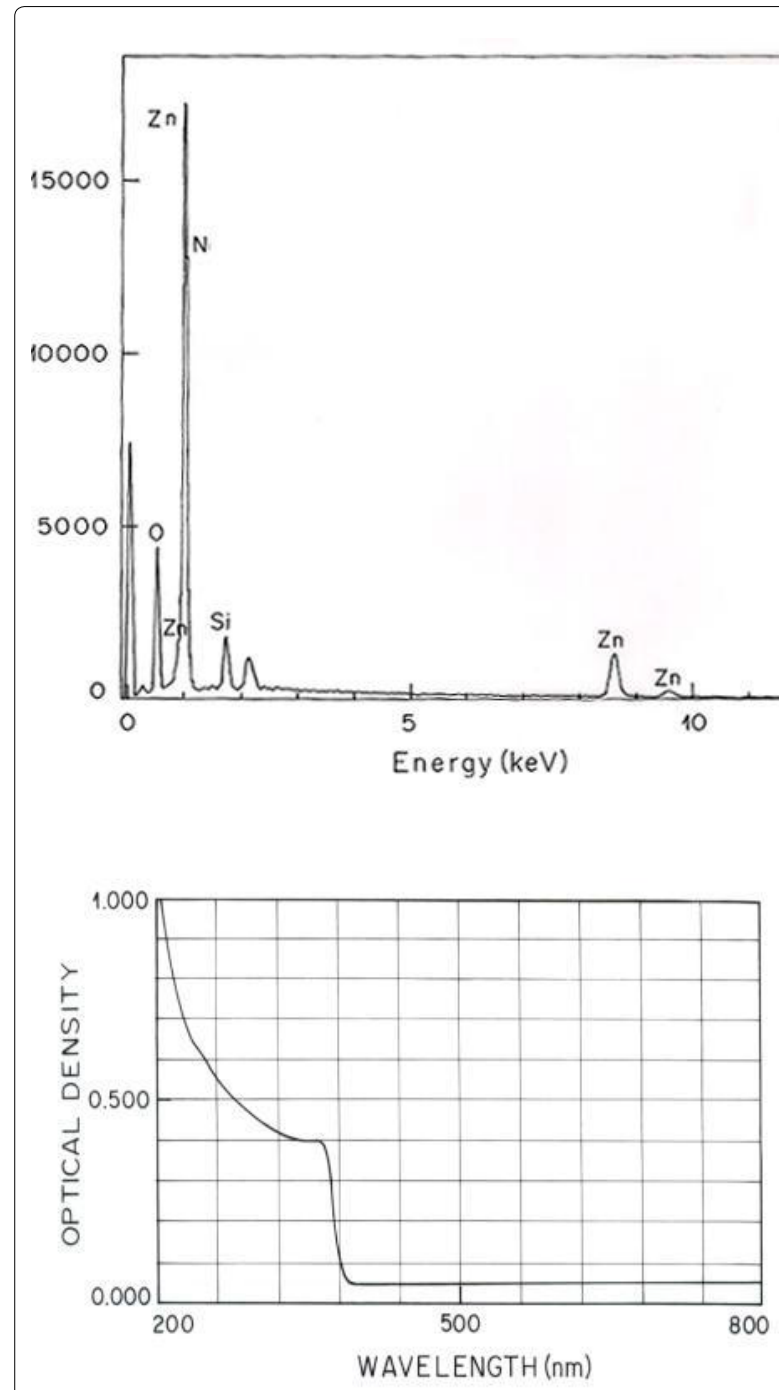

Figure 3: EDX and UV-VIS spectra of ZnO nanoparticles. is initiated by the dehydration to $\mathrm{C}_{2} \mathrm{H}_{4}$. But the selectivity for the two reactions is initiated by the acid-base properties of the oxide surface. This behavior of the sensors is attributed to the increase of concentration of oxygen species on the surface by replacing adsorbed hydroxyl groups and conversion of adsorbed oxygen species by following reactions: $\mathrm{O}_{2}($ gas $) \rightarrow \mathrm{O}_{2}($ ads $) \rightarrow 2 \mathrm{O}-($ ads $) \rightarrow 2 \mathrm{O}_{2}-$

\section{TEM analysis}

Transmission electron microscope examination can yield the information like topography, morphology, composition as well as crystallographic information's (Figure 5).

In TEM there is no change in the refractive index of the medium when the illumination beam is deflected, the vacuum in the lens is the same as the vacuum in the column.

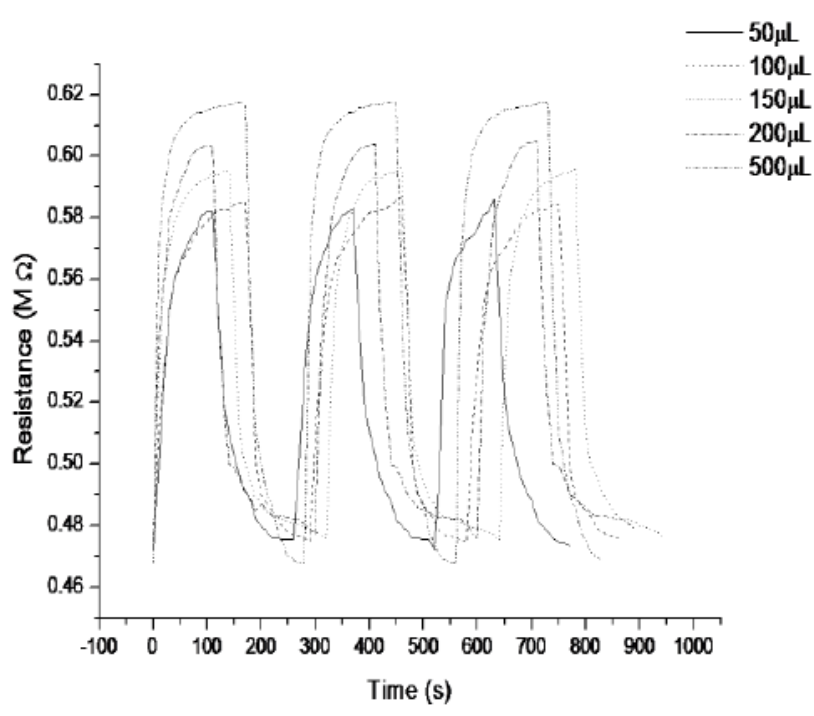

Figure 4: A graph showing resistance versus time for ethanol vapour sensors.

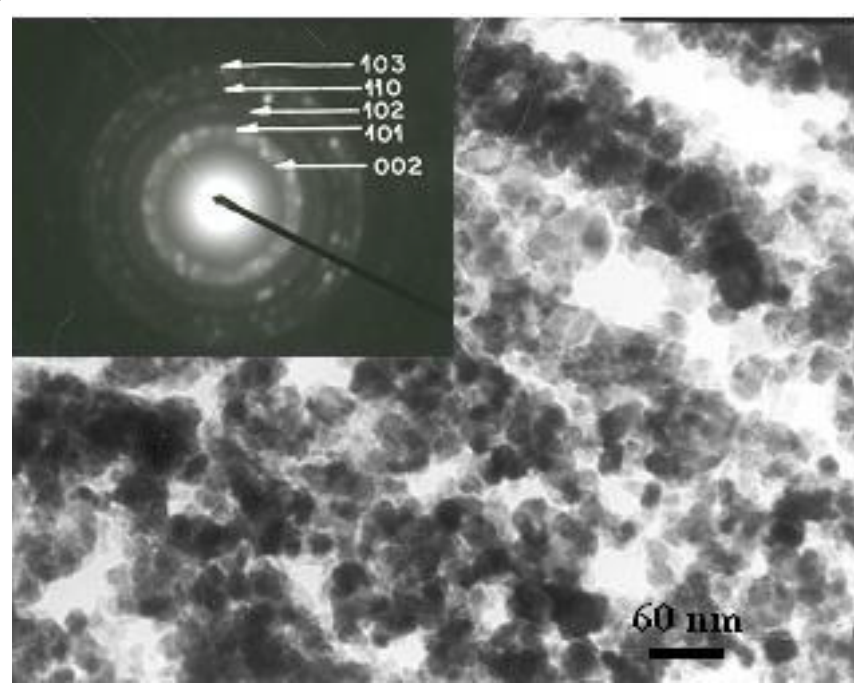

Figure 5: TEM images and electron diffraction patterns of $\mathrm{ZnO}$ nanoparticles at different temperatures.

This image reveals that the product consist of spherical particles with the average size of $5-20 \mathrm{~nm}$. 


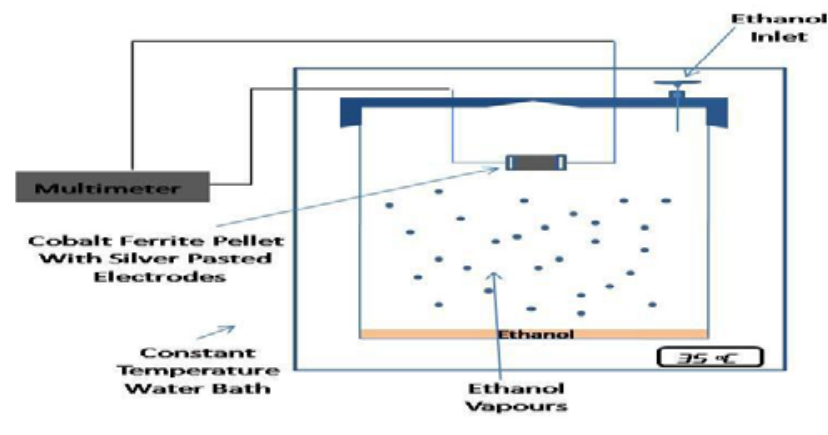

Figure 6 : Ethanol vapor sensor setup.

\section{Analysis of ethanol vapour}

The entire setup for measuring sensitivity of the ethanol vapour sensor is shown in Figure 6. The resistance of the sensor is measured, with respect to time upto the saturation of the sensor to the gas, and then the air was allowed into the chamber. The resistance measurement of the sensor is continued up to the complete recovery of the sensor resistance to the air ambient value. The measurement was repeated for number of cycles to determine the cyclic nature of the sensor. The resistance of the sensor is measured for different temperatures in the range of $25^{\circ} \mathrm{C}$ to $45^{\circ} \mathrm{C}$ at an interval of $5^{\circ} \mathrm{C}$ under an ethanol concentration of $25.02,50.05,75.08,100.11$ and 150.17 $\mathrm{ppm}$. in ethanol vapor-air ambient. Gas response (S) is defined as the ratio of the change in resistance of the sensor on exposure to the target gas to the original resistance in air.

\section{Conclusion}

In this study, the structures and sensing properties of $\mathrm{ZnO}$ nanoparticles thin films as an ethanol vapor gas sensor obtained by characterization were investigated. XRD, TEM, EDX and UV visible characterization reveals surface morphology and particles size of $\mathrm{ZnO}$ nanoparticles. The film exhibited good sensitivity to the ethanol vapors with quick response-recovery characteristics, and it was found that the sensitivity and selectivity is increased $\mathrm{ZnO}$ nanoparticles seems to be a promising semiconducting material for the detection of ethanol vapor. The change in electrical resistance was used as the measure of ethanol vapor response at different temperatures in the range of $25^{\circ} \mathrm{C}$ to $45^{\circ} \mathrm{C}$ at an interval of $5^{\circ} \mathrm{C}$ under an ethanol concentration of $25,50,75,100$ and $150 \mu$. These results showed that the indium zinc oxide film based ethanol sensor has high response quick recovery time and selectivity to the ethanol vapor.

\section{Acknowledgements}

We are thankful to Prof. R.C. Budhani, Director, CSIR-National Physical Laboratory, Delhi, India for permitting to carry out and publishing this work.

\section{References}

1. Baxter JB, Aydil ES (2005) Appl Phys Lett 86.

2. Huang $\mathrm{MH}$, Mao S, Feick $\mathrm{H}$, Yan $\mathrm{H}$, Wu $\mathrm{Y}$, et al. (2001) Room-temperature ultraviolet nanowire nanolasers. Science 292: 1897-1899.

3. Song J, Zhou J, Wang ZL, Wang X, Liu J, et al. (2006) Piezoelectric Field Effect Transistor and Nanoforce Sensor Based on a Single ZnO NanowireNano Lett 6: 2768-2772.

4. Wang ZL (2004) Functional oxide nanobelts: materials, properties and potential applications in nanosystems and biotechnology. Annu Rev Phys Chem 55: 159-196.
5. Sawai J, Igarashi H, Hashimoto A, Kokugan T, Shimizu M, et al. (1996) J Chem Eng Japan 29: 627-633.

6. Li Q, Kumar V, Li Y, Zhang H, Marks TJ, et al. (2005) J Am Chem Soc 17.

7. Barua S, Pal SK, Dutta J (2012) Nanostructured Zinc Oxide for Water Treatment. Nanoscience and Nanotechnology-Asia 2: 90

8. Webb JB, Williams DF, Buchanan M (1981) Trans- parent and Highly Conductive Films of ZnO Prepared by RF Reactive Magnetron Sputtering. Appl Phys Lett 39: 640-642.

9. Kang SJ, Choi JY, Chang DH, Yoon YS (2005) A Study on the Growth and Piezoelectric Characteristics of ZnO Thin Film Using a RF Magnetron Sputtering Method. Journal of Korean Physics Society 47: S589-S594.

10. Chakrabarti S, Dutta BK (2004) Photocatalytic degradation of model textile dyes in wastewater using $\mathrm{ZnO}$ as semiconductor catalyst. J Hazard Mater 112: $269-278$.

11. Zak AK, Razali R, Majid WH, Darroudi M (2011) Synthesis and characterization of $\mathrm{ZnO}$ nanoparticles prepared in gelatin media. Mater Lett 65:70-73.

12. Zak AK, Abrishami ME, Majid WHAbd, Yousefi R, Hosseini SM (2011) Effects of annealing temperature on some structural and optical properties of $\mathrm{ZnO}$ nanoparticles prepared by a modified sol-gel combustion method. Ceram Inter 37: 393-398.

13. Wang Y, Zhang C, Bi S, Luo G (2010) Preparation of ZnO nanoparticles using the direct precipitation method in a membrane dispersion micro-structured reactor. Powder Technol 202: 130-136.

14. $\mathrm{Lu} \mathrm{CH}$, Yeh $\mathrm{CH}(2000)$ Influence of hydrothermal conditions on the morphology and particle size of zinc oxide powder. Ceram Inter 26: 351-357.

15. Sangkhaprom N, Supaphol P, Pavarajarn V (2010) Fibrous zinc oxide prepared by combined electrospinning and solvothermal techniques. Ceram Inter 36: 357-360.

16. Yousefi R, Muhamad MR, Zak AK (2010) Investigation of indium oxide as a self-catalyst in $\mathrm{ZnO} / \mathrm{ZnInO}$ heterostructure nanowires growth. Thin Solid Films 518: 5971-5977.

17. Ivanov P, Llobet E, Vilanova X, Brezmes J, Hubalek J, Correig X (2004) Development of high sensitivity ethanol gas sensors based on Pt-doped $\mathrm{SnO} 2$ surfaces. Sens Actuators B 99: 201-206.

18. Paraguay DF, Miki-Yoshida M, Morales J, Solis J, Estrada LW (2000) Influence of $\mathrm{Al}, \mathrm{In}, \mathrm{Cu}, \mathrm{Fe}$ and $\mathrm{Sn}$ dopants on the response of thin film $\mathrm{ZnO}$ gas sensor to ethanol vapor. Thin Solid Films 373: 137-140.

19. Xu CH, Lui HF, Surya C (2011) Synthetics of $\mathrm{ZnO}$ nanostructures by thermal oxidation in water vapor containing environments. Mater Lett 65: 27-30.

20. Islam MR, Kumazawa N, Takeuchi M (1999) Chemical sensor based on titanium dioxide thick film:Enhancement of selectivity by surface coating. Appl Surf Sci 142: 262-266.

21. Matsushima S, Maekawa T, Tamaki J, Miura N, Yamazoe N (1989) Role of Additives on Alcohol Sensing by Semiconductor Gas Sensor. Chem Lett 18: $845-848$.

22. Kohl D (1989) Surface processes in the detection of reducing gases with SnO2based devices. Sens Actuators 18: 71-113.

23. Pandya HJ, Chandra S, Vyas AL (2011) MEMS-based Ethanol Sensor Using Zinc Oxide Nanostructured Films. SENSORDEVICES: The Second International Conference on Sensor Device Technologies and Applications.

24. Tyagi S, Devi S, Paul AK (2014) Synthesis and characterization of zinc oxide nanoparticles for ethanol detection. Environmental Science and Engineering 707-708. 